$\begin{array}{ll}\text { Variants } & \begin{array}{l}\text { Variants } \\ \text { The Journal of the European Society for Textual } \\ \text { Scholarship }\end{array}\end{array}$

15-16 | 2021

Textual Scholarship in the Twenty-First Century

\title{
Reflections on Digital Scholarly Editions: From Humanities Computing to Digital Humanities, the Influence of Web 2.0, and the Impact of the Editorial Process
}

Michelle Doran

\section{(2) OpenEdition \\ Journals}

Electronic version

URL: https://journals.openedition.org/variants/1414

DOI: 10.4000/variants. 1414

ISSN: 1879-6095

Publisher

European Society for Textual Scholarship

Printed version

Date of publication: 1 July 2021

Number of pages: 213-230

ISSN: 1573-3084

Electronic reference

Michelle Doran, "Reflections on Digital Scholarly Editions: From Humanities Computing to Digital Humanities, the Influence of Web 2.0, and the Impact of the Editorial Process", Variants [Online], 15-16 | 2021, Online since 01 July 2021, connection on 16 July 2021. URL: http://journals.openedition.org/ variants/1414; DOI: https://doi.org/10.4000/variants. 1414 


\title{
Reflections on Digital Scholarly Editions: From Humanities Computing to Digital Humanities, the Influence of Web 2.0, and the Impact of the Editorial Process
}

Michelle Doran

\begin{abstract}
This essay offers an ongoing process of reflection on the role of the digital medium in creating scholarly editions of medieval Irish texts. What are the relationships between (digital) textual scholarship, (digital) scholarly editing, humanities computing, and Digital Humanities? How have these concepts changed our editorial praxis? The author discusses four different digital scholarly editions of medieval Irish manuscripts and then focuses one specific case: @ChronHib's "The Poems of Blathmac Tweets". This Twitter account of the eponymous edition presents a stimulating example of new forms of (user) engagement and the role of editors and audiences in the digital realm.
\end{abstract}

In 2016, Joris J. Van Zundert wrote of digital scholarly editions (DSEs) that "they are a far cry from what many expected them to be" (Van Zundert 2016b. 899). The reason for this, Van Zundert suggests, is what he labels "paradigmatic regression", that is "the social shaping of a technological interface such that it can no longer express essential properties of an intended paradigm" (Van Zundert 2016b. 901). The central thesis of Van Zundert's work is that the successful exploration of the potential of the DSE can only be achieved if we can cease the process of reverting to existing paradigms when attempting to build new models. In a further contribution, he contends that an additional limiting factor is that textual scholars remain deeply entrenched in the "ideal of publishing the definitive edition" (Van Zundert 2018, 5: 12). He continues, "[m] ost editors think of an edition as a complete and finished product - something that should not be tampered with, because it is argued and polished with arduous effort to academic perfection" (ibid.).

In this essay, I follow a similar line of enquiry to Van Zundert, asking if existing definitions of DSEs have similarly served to limit the potential for textual scholars to reimagine scholarly editing in the digital age. To put it another way, are we overly concerned with what these resources should be, rather than what they could be? This is not a new question (see e.g. Prescott 2003. Robinson 2013. 106 and Pierazzo 2014. 210), but I hope to offer a different perspective. This essay takes the position that scholarly editing in its digital format belongs 
to the wider area of digital humanities and as such it is shaped not only by the underlying digital medium but also by the social, methodological, and epistemological commitments of the field which has developed to support digital scholarship in the humanities. Despite a history spanning over seventy years, digital humanities is regularly defined as being in a continual state of transition. It follows that the scholarly outputs which result from activities within the field must continue to reflect the nature of the practice as it evolves. Therefore, rather than emphasising the ongoing influence of the print paradigm, I suggest that earlier iterations of digital humanities (generally referred to as humanities computing) may be having an equally delimiting effect on the new models of scholarly editing in the digital format.

To achieve this, the present essay is divided into four main sections. The first part provides some contextual remarks regarding the developmental phases of digital humanities and the influence of Web 2.0 technologies on the field as it is currently conceived. I will then move on to discuss recent debates regarding the concept of the social edition as it manifests in the digital format. In light of this discussion, the third part reviews the recent series of Tweets transcribing and translating the Early Irish poetic text, The Poems of Blathmac on the Chronologicon Hibernicum Twitter feed (@ChronHib). Whilst from a technical perspective this work falls far short of the desideratum of a digital edition, it remains an outstanding example of many of the editorial ideologies which underlie them 1 How, then, should we evaluate the digital output of the Chronologicon Hibernicum Twitter feed? Is it necessary at the current time for a digital edition to espouse both the technical and the ideological best practices set forth by the digital editing community? How might our desire to legitimize our work limit its potential? The final section addresses these questions. However, before moving to the main arguments of the essay, some contextual remarks regarding the development of this piece seem appropriate.

This essay is best-described as the result of an unintended iterative research process. It began as a chapter of my doctoral thesis, submitted in early 2015 though written over more years than I care to recount. The primary subjects of this work were the ideological and epistemological perspectives which have

1 As an example of the discourse regarding the desideratum of a digital edition, we might consider the contribution of Marina Buzzoni 2016 For Buzzoni, the datafication of the scholarly object is key to determining which features should be defined as fundamental to a digital edition. Buzzoni lists five features, or domains, which a digital scholarly edition should present, "regardless of the subtype to which it belongs" (Buzzoni 2016. 61). In other words, regardless of the scholarly purpose of the edition, a digital edition must be structured in accordance with certain digital functionalities and the intention of these functionalities is to fully exploit the digital environment. These domains are listed as: scalability, relationability, interoperability, multimediality and multimodality, and lastly, interactivity. According to Buzzoni, a protocol for digital scholarly editions is a desirable means of testing the editorial product through its core functionalities, "provided that the protocol encompasses the domains in which scholarly digital editions may offer important advantages over paper editions, without being too strict as to orient the editor to follow a specific ecdotic praxis"(Buzzoni 2016, 81). 
informed much of the way in which medieval Irish texts are rendered into modern editions and the aim of the chapter in question was to examine the role of the digital medium in the production of editions of medieval Irish texts. Here, I concluded that " $[\mathrm{t}] \mathrm{o}$ date, the course of digital humanities computing in medieval Irish scholarly editing demonstrates the willingness of certain scholars to engage with the theories, methodologies and technologies of contemporary textual criticism"(Doran 2015, 337). In late 2016, I had the opportunity to revise this chapter as part of my participation in the MA in Digital Humanities at An Foras Feasa, National University Maynooth. Recognising my error in nomenclature, I wrote that "my previous assertion presupposes that Digital Humanities and humanities computing are synonymous [...] this is not the case. The implementation of the tools of humanities computing does not necessitate the implementation of the epistemology of Digital Humanities". I continued that:

A more accurate assessment of the current state of affairs might read, to date, the course of humanities computing in medieval Irish scholarly editing demonstrates the willingness of certain scholars to engage with the methodologies and technologies of contemporary textual criticism. However, the ideologies of Digital Humanities and the epistemic commitments of the Web 2.0 model have largely been ignored.

(Doran 2016)

In 2018, some of my arguments found a home in the recently published Digital Technology and the Practices of Humanities Research (Edmond 2020). Once again, I found myself revising previous conclusions, this time as a result of a series of Tweets translating and transcribing the Early Irish poetic text The Poems of Blathmac on the Chronologicon Hibernicum Twitter feed. In this piece, I reversed the gaze of my research and used the@ChronHib Tweets as a means of examining the limitations imposed on the editorial process by the various defining criteria of the digital scholarly edition (DSE), that is, the editorial product. However, as a result of the arrival of my son in May 2019, I was unable to participate in the final tranche of revisions. This current essay combines each of these three pieces, doubtless it will be revisited.

I offer these preliminary remarks because to my mind they highlight much of what is entailed in textual scholarship in the twenty-first century. Firstly, it is an iterative and self-conscious research process, indeed a desire for processorientated evaluation criteria is coming increasingly to the fore and was a major motivating factor in my reconsideration of the concept of the DSE in light of the@ChronHib Twitter feed which will be discussed in further detail presently. As Elena Pierazzo maintains, "[d]igital editing as research may still produce digital editions, but such editions may be considered just one of the outcomes of the research, the embodiment of a new editorial theory or model, and not its most important outcome" (Pierazzo 2014. 217). Of course, not all contemporary 
textual scholarship is concerned with the production of a DSE, but it would be difficult, if not impossible, to deny the extent to which the specific medial changes of new technologies have contributed to changing epistemologies in the wider field of textual scholarship: "we shape our tools, and thereafter our tools shape us" (Pierazzo 2016, 3). Editorial practices are now located along a continuous spectrum of digital, predigital and nondigital scholarship. In this context, tensions emerge from "the multidisciplinary methodological interaction that has arisen to support the theoretical and practical development of the digital scholarly edition over the recent years" (Van Zundert 2016a. 83). This essay is primarily concerned with four such interactions, that of (digital) textual scholarship, humanities computing, digital humanities and Web 2.0 technologies.

The developmental stages of digital humanities are broadly conceived as being characterized by two transformative phases, often separated by a discursive shift in identity from humanities computing to digital humanities. Precisely when this transition occurred is difficult to pinpoint. According to correspondence between John Unsworth and Matthew Kirschenbaum reported by the latter (Kirschenbaum 2010, 56-57), the term originates from a conversation held in November 2001 regarding the title of the highly-influential Blackwell 2004 Companion to Digital Humanities (Schreibman et al. 2014). By 2009, Patrick Svennson observed that whilst the use of the term humanities computing was still frequent there was an increased use of digital humanities (relative to humanities computing). In the same contribution, Svennson argued that the discursive shift from humanities computing to digital humanities represented more than just a repackaging of the former. He continued that humanities computing, with its focus on "the instrumental, methodological, textual and digitalized", carries with it a set of epistemic commitments and conventions that are not necessarily compatible with digital humanities as it was and continues to be conceived (Svensson 2009). Interestingly, the tools and platforms of the Web 2.0 model do not play a dominant role in Svensson's notion of digital humanities.

A decade after Tim Berners-Lee's initial proposal for the World Wide Web, Darcy DiNucci coined the term "Web 2.0", a concept which describes the shift from the static webpages of the first iteration of the Web to "the web as platform" model, the emergence of prosumers (a blending of the roles of producers and consumers) and the large scale development of social networking software (DiNucci 1999, 32). In 2008, drawing on the popularized labels Web 1.0 and Web 2.0, Cathy Davidson identified two phases of digital humanities, Humanities 1.0 and Humanities 2.0. Here, Davidson aligns the Humanities 1.0 with the large-scale digitising projects of humanities computing, she continues that "Humanities 2.0 is distinguished from monumental, first-generation, data-based projects not just by its interactivity but also by openness about participation grounded in a 
different set of theoretical premises, which decenter knowledge and authority" (Davidson 2008, 711-712). There is a clear assumption of the transformative nature of Web 2.0 epistemologies within the humanities in Davidson's manifesto. The change envisioned extends far beyond how we research and who can research and moves to redefine the humanities more generally: "Additional concepts decentred by Web 2.0 epistemologies include authorship, publication, refereeing, collaboration, participation, customizing, interdisciplinarity, credentialing, expertise, norms, training, mastery, hierarchy, taxonomy, professionalism, rigor, excellence, standards and status" (Davidson 2008, 712). It is also noteworthy that Davidson does not envision an end goal for Humanities 2.0, there is no product, only process.

The differentiation (or lack thereof) between humanities computing and digital humanities may appear at first to be a red herring. However, when one considers the shared history of digital textual scholarship and humanities computing, the assimilation of humanities computing into a big tent digital humanities fundamentally affects the landscape of textual scholarship in the twenty-first century. In the field of digital humanities, there exists a large corpus of research literature that attempts to delineate and define DSEs. However, much of this scholarship was developed prior to the discursive shift from humanities computing to digital humanities and the ubiquity of the Web 2.0 model with which we are now familiar and therefore does not accurately reflect the full range of useful possibilities present for academic engagement and interaction around their research objects. Despite this, these resources of the "pioneer era" of digital scholarly editing remain fundamental to our understanding of what constitutes a DSE (Robinson 2005). When Svensson's observations are considered alongside the Humanities 2.0 model proposed by Davidson tensions arise. In a further contribution on the landscape of digital humanities published in 2010. Svensson writes that humanities computing will not become the Humanities 2.0 envisioned by Davidson, that even those scholars deeply engaged in humanities computing might not identify as "digital humanists". As Svensson points out, there is a risk that different epistemic traditions and goals are conflated in the reading of humanities computing as digital humanities (Svensson 2010). In what follows, I suggest that the editorial products of digital textual scholarship often continue to align with the humanities computing model, whereas the underlying ideological commitments of the social view of digital editing reflect a greater cognisance of the epistemic cultures of digital humanities and Web 2.0.

\section{II}

From the perspective of Early and Medieval Irish Studies, there are a number of outstanding examples of the "monumental, first-generation, data-based projects" of humanities computing (Davidson 2008, 711-712). A prominent example would be Corpus of Electronic Texts (CELT), "Ireland's longest running Humanities Computing project" (1997-). It currently constitutes the digital text of 1636 
documents from the medieval through to the modern period in Irish, Latin, Anglo-Norman French and English. These texts may be read online and are also available in HTML, SGML and XML formats. The SGML/XML files are encoded following the TEI Guidelines (TEI Consortium 2020). Texts are taken from "the best printed editions" and in a method of presentation common at the time the project started, the digital text constitutes the main body of the edition without the critical apparatus ${ }^{2}$ Consequently, resources such as CELT have been surrounded by debates regarding the appropriateness of the label edition with "project", "archive", "thematic research collection", "database", "knowledge site", "digital edition 2.0" and even "arsenal" all being suggested as alternatives 3

Discussions regarding the critical nature of these editions (or the lack thereof) often overlook the arguments regarding text, edition and the role of technology inherent in the means, methods, models and media of presentation. Digital iterations of scholarly editions, in fact, often deliver applications that represent electronic versions of predigital practices, and with these applications the underlying ideologies of the inherited legacies are replicated and consequently redistributed. For example, text encoding and markup are two core components of humanities computing. In 2002, Koenraad de Smedt wrote that " $t \mathrm{t}] \mathrm{ext}$ encoding seems to create the foundation for almost any use of computers in the humanities" (qtd. in Svensson 2016, 66). His comments regarding the manner in which these technologies are applied, representing an instrumental and uncritical approach to humanities computing are as relevant today as they were in 2002. Regarding the development of SGML into TEI-XML descriptive standard, Van Zundert argues that the scholarly editing community "turned hypertext markup into a descriptive model of the book, and we have produced digital book metaphors ever since" (Van Zundert 2016b).

Resources such as CELT are firmly grounded in the epistemic commitments of humanities computing foregrounding the two fundamentals of the field as articulated by Svensson: "computer as instrumental tool" and "text as object" (2009). As manifestations of humanities computing practice, they may not be compatible with those formulations of the editorial process which emphasize the role of the tools of social networking and social media in blurring the boundaries of the traditional editorial paradigm which reflect a greater cognisance of the epistemic cultures of digital humanities and Web 2.0 technologies. That is not to suggest that they do not constitute DSEs or that DSEs modelled on the epistemic traditions of humanities computing are no longer valid. In fact, I am suggesting that the reverse may be the case. These resources foreground our understanding of what constitutes a DSE and therefore, the risk to digital textual scholarship in conflating these approaches is that the epistemological biases of the humanities

2 See the "About Us" page of CELT: https://celt.ucc.ie/about.html

3 For a discussion of the terms employed to describe "large-scale text-based electronic scholarship" and of their implications for digital textual scholarship, see Price 2009 
computing model will limit our understanding of how the digital is impacting upon textual scholarship by excluding from the conversation those resources which do not constitute DSEs in accordance with this model.

An illustrative example of the tensions arising between the different epistemic traditions involved in textual scholarship in the twenty-first century is the concept of the digital social edition. In 2012. Ray Siemens along with several others presented a preliminary model of the social edition in its electronic form as part of a basic typology of the scholarly electronic edition. Here, the authors identify four types of editions based on the approach that the creators of each type take when handling and engaging with the textual materials. First, there is a dynamic text which consists of the edited electronic text plus analytical tools for its readers. Next, the hypertext edition which presents the text together with supporting materials in digital form to aid reader navigation and subsequent analysis. Following this is the dynamic edition, of which the authors note there is not yet an exemplary touchstone. The dynamic edition is an electronic text augmented by dynamic analytical means, hypertextual links to fixed resources together with means of identifying and collaborating with external resources.

The last type, the social edition, remains undefined. However, Siemens et al. identify numerous characteristics of the "definitive social edition", which will be discussed in greater detail presently. The authors suggest that the order in which these different editions are set forth represents a sort of chronology for the development of digital scholarly editing and they opine that the social edition is representative of the most recent developments in editorial theory (Siemens et al. 2012. 446-48). At the core of the discussion of the social edition are the tools which enable the model: "such tools facilitate a model of textual interaction and intervention that encourage us to see the scholarly text as a process rather than a product, and the initial, primary editor as facilitator, rather than progenitor, of textual knowledge creation" (Siemens et al. 2012, ibid). These tools are the new and emerging, scholarly and non-scholarly digital interactive tools of social media and the Web 2.0 model of the World Wide Web with which we are now familiar.

An awareness of the ideas set forth by Davidson and her model of Humanities 2.0 discussed earlier in this essay are crucial to our understanding of the social edition described by Siemens and his collaborators. First, both contributions are predicated on the assimilation of the tools of Web 2.0 by the practitioners of digital textual scholarship. Secondly, the democratization of knowledge and authority envisioned by Davidson is evident in the description of the social edition where it is maintained that the integration of social media tools can push the boundaries of authority, "shifting power from a single editor, who shapes the reading of any given text, to a group of readers comprising a community whose interpretations themselves form a new method of making meaning out of the material" (Siemens et al. 2012, 453). In their estimation, the definitive social edition eschews traditional, hierarchical models of scholarly discourse in favour of a plurality of community-generated authorities. As with Davidson's model of 
Humanities 2.0, the proponents of the social edition privilege process over end result (Siemens et al. 2012 ibid). The sequential presentation of both arguments is not unproblematic, the assumption is that there is only one approach feasible at any given time, that the most recent formulation of an academic field supersedes the one that came before it. Accordingly, the Humanities 2.0 model displaces the Humanities 1.0 and the emerging social edition represents the most recent developments in the theories of digital textual scholarship.

The model of the social edition presented by Siemens and his coauthors, is only one characterization of the role of the social view of texts in digital textual criticism. In an article canvassing the multiple meanings of the word "social" as it is applied to editions, editing and texts in the field of digital textual scholarship, Robinson argues that we should be particularly conscious of how we employ the label in each of these specific contexts. Many, if not all, of Robinson's criticisms of the model are levelled against the Social Edition of the Devonshire Manuscript by Siemens and his collaborators and the associated scholarship including their 2012 article. Citing the work of Jerome McGann and Donald McKenzie, which underpins Siemens' social edition, Robinson remarks that whilst the social view of texts has been influential in many areas, the "social text edition" has not yet come to fruition. He continues that many of the projects which claim to be "social editing" projects are transcription projects which enable editing projects and not editing projects in and of themselves. Here, he observes that many social editing projects continue to uphold the traditional editor-driven model of textual criticism: documentation is selected on the basis of editorial requirements; external contributions are closely monitored by a single editor or more likely a team of editors and contributors are excluded from stages of the project. He posits that the recent enthusiasm surrounding the word "social" in the context of digital textual scholarship has led to "unrealistic expectations invoked by misapplication of the term". Once again, the juxtaposition of editorial products (social text editions and social editions) and editorial processes (social editing) is evident throughout Robinson's animadversions of the social edition as set forth by Siemens and his coauthors. The former, he concludes, do not exist. The latter is a misleading misnomer which detracts from the possibilities offered by the digital medium. In his estimation, these possibilities are "in the making of editions that are more inclusive in their making, which achieve a wider impact and create new understandings in expanding circles of readership, whether or not we choose to label these as social" (Robinson 2016b).

Of course, the model set forth by Siemens et al. is not the only formulation of the integration of social technologies into textual scholarship. Other scholars have attempted to create a broader definition. For example, based on his extensive work collecting and cataloging DSEs, Patrick Sahle presents his defining criteria as four questions. In brief, these questions are: 1) Is there a full representation of the subject in question?; 2) Is it critical?; 3) Is the edition of academic quality?; and 4) Does the edition follow a digital paradigm? (Sahle 2016. 38). According to Sahle, "Scholarly digital editions are scholarly editions that are 
guided by a digital paradigm in their theory, method and practice" (Sahle 2016. 28). In this context, a scholarly edition is the "critical representation of historic documents" (Sahle 2016, 23). Sahle distinguishes between digitized or scanned print editions which continue to uphold the print paradigm and digital editions, writing that the latter cannot be printed without a significant loss of information and functionality. Undoubtedly, the former may replicate some of the features of DSEs - such as search functions - but they do not themselves constitute such an edition. According to Sahle, the digital paradigm subverts, inverts and reverses many of the processes and assumptions of the traditional editorial paradigm. Though not explicitly referenced, the influence of the concepts of Web 2.0 and Humanities 2.0 as digital humanities is evident throughout Sahle's description of the digital paradigm in editing, key characteristics of which are "multimedia", "hypertexts", "hyperlinks", "fluid publication", "process rather than product", "collaboration", "a strong tendency towards multiple texts", "a pluralistic notion of text" and "transmedialization". Once again, the concept of social editing comes into play with contributions coming from external institutions as well as "communities of the scholarly or even wider interested public" (Sahle 2016, 30).

Although brief, these examples of the discussions surrounding the (digital) social edition highlight many the tensions which can result from "the multidisciplinary methodological interaction that has arisen to support the theoretical and practical development of the digital scholarly edition over the recent years" (Van Zundert 2016a, 83). We see throughout the debate efforts to identify and separate overlapping epistemologies and to articulate what is truly revolutionary about DSEs. Where supporters point to the novelty of the social edition in its digital format, critics respond that the social concept of text was founded in predigital textual scholarship. The challenges expressed here are not necessarily new, nor are they unique to the social edition. Pierazzo makes similar observations about the role of variation in digital editions (Pierazzo 2016, 45-46) and the same has been said about the ability for DSEs to display images of the original document (Robinson 2016a. 181-183). Of course, all of this brings us back to the role of the "book metaphor" in digital textual scholarship as articulated by Van Zundert and the formalization of these practices through the articulation of humanities computing as digital humanities. Recent proponents of the DSE have embraced the current state of affairs, emphasising the impact of the digital editorial process over the digital editorial product. Whilst it is certainly true that the social concept of text has its foundations in predigital editorial theory, the manner in which this is achieved is radically different in digital editorial practice. In a piece discussing variation and the DSE, Pierazzo writes that "The digital environment in its various embodiments may well be a more flexible space in which to seek public engagement with editorial endeavours, if indeed editors are willing to do it" (Pierazzo 2016, 55). I will conclude by considering one such example. 


\section{III}

Chronologicon Hibernicum - A Probabilistic Chronological Framework for Dating Early Irish Language Developments and Literature (ChronHib) is a research project funded by the European Research Council under the HORIZON 2020 Framework Programme between September 2015 and August 2020. The project which is hosted by National University of Ireland Maynooth, aims to refine the methodology for dating Early Medieval Irish language developments (ca. sixth to mid tenth century AD) and to build a chronological framework of linguistic changes that can then be used to date literary texts within the Early Irish period. This will be achieved through the linguistic profiling of externally dated texts and by using statistical methods for the serialization of data, and for estimating dates using Bayesian inference.

The project maintains an active social media presence with the dual intention of propagating the project results and raising awareness of the Early Irish language and its importance for the intellectual history of Ireland and Europe. As of March 2020, the project's Twitter account (@ChronHib) posted over 11,700 Tweets loosely related to the themes of research on Early Irish language and literature and has almost 2,900 followers. The account administrator, David Stifter, is a professor of Old Irish at National University of Ireland Maynooth and is Principal Investigator on the ChronHib project. In January 2017, Stifter began posting a series of Tweets transcribing and translating the eighth century Old Irish Poems of Blathmac mac Con Brettan maic Conguso do Feraib Rois, "Blathmac, Son of Cú Brettan, son of Congus of the Fir Rois" (The Poems of Blathmac; see Figure 11. The stated intention of the Tweets was to create an outlet for a preliminary version of an edition of the poems and comments were requested from the account's followers.

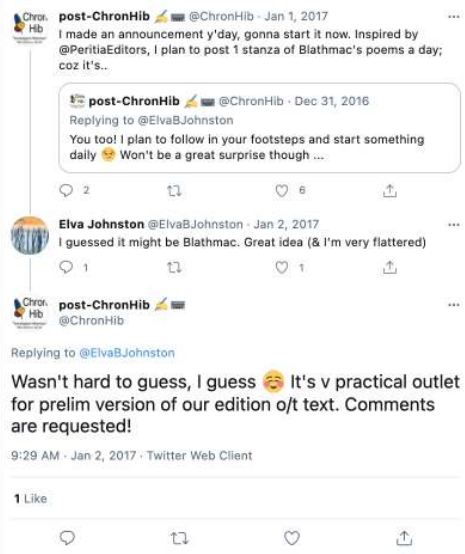

Figure 1: The Poems of Blathmac Twitter series announcement.* 
The work took place from January to October 2017 and constitutes the presentation of 303 four-line stanzas, roughly one stanza per day. In general, the Tweets follow the same format. First, a Tweet containing an image of the relevant section of the manuscript is posted together with the stanza number and a semi-diplomatic transcription of the text, while editorial notes regarding the transcription are added in the comments section (see Figure 2). Next, the translation of the stanza is posted across two Tweets both containing the stanza number and a translation of two lines of the relevant transcription together with modern iconography relating to the translation - again, with editorial notes entered in the comments section (see Figure 3).

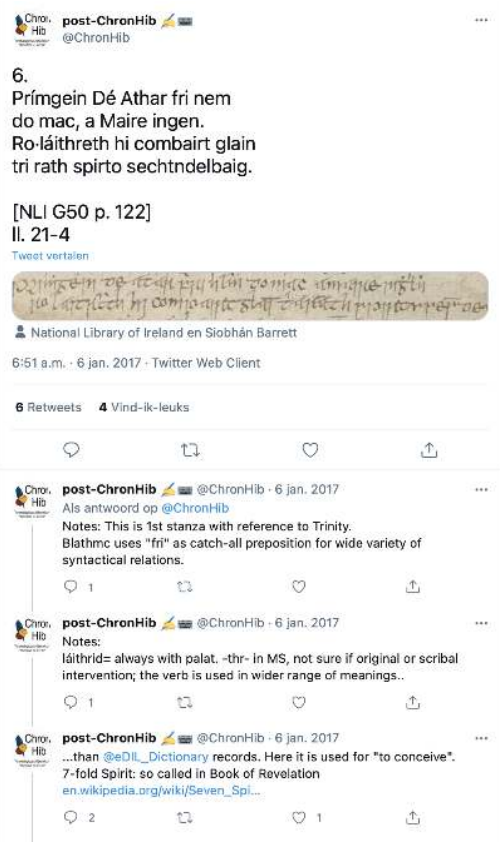

Figure 2: @ChromHib tweets regarding "The Poems of Blathmac", stanza 6: transcription and comments. ${ }^{\dagger}$

The comments section opens up the editorial methodology in a number of ways. Firstly, it allows insight into the decision-making process in a way that traditional print editions, or indeed many DSEs do not. Consider for example

* Twitter thread available via: https://twitter.com/ChronHib/status/8158 37431735943173 (accessed 2 July 2021)

† Twitter thread available via: https://twitter.com/ChronHib/status/8172 47306697216000 (accessed 2 July 2021). 
2. Proch post-ChronHib $\angle=$

6.

The first-born of God, the Father, in heaven

(is) your son, Virgin Mary.

(1/2; to be cont.)

[The Trinity; $\odot$ Douglas Blanchard]

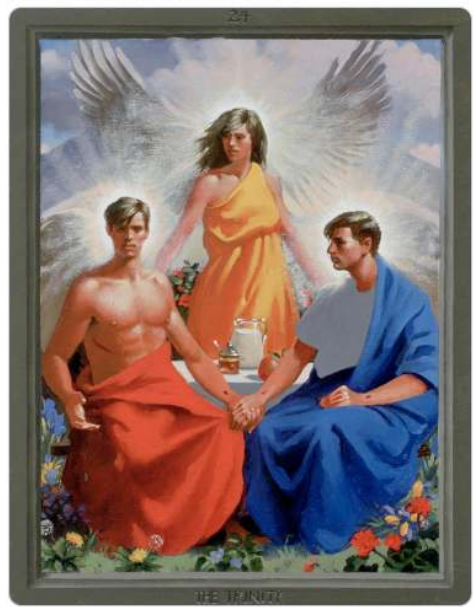

7:02 AM - Jan 6, 2017 - Twitter Web Client

2 Retweets 1 Quote Tweet 6 Likes

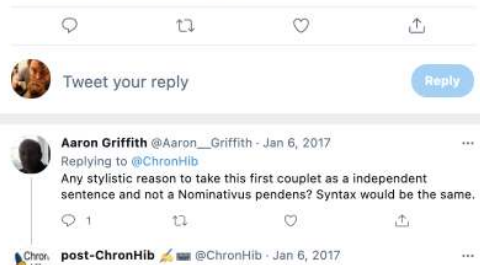

Crvor. post-ChronHib $2 \equiv$ Ho
No, you could understand it that way. But frequ. the 2 couplets are separated by syntactic break; l assumed this here, too.

(a)

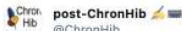

6. (cont.)

$\mathrm{He}$ has been bestowed in pure conception

through the grace of the Septiform Spirit.

$(2 / 2)$

[the 7 gifts of the Holy Spirit]

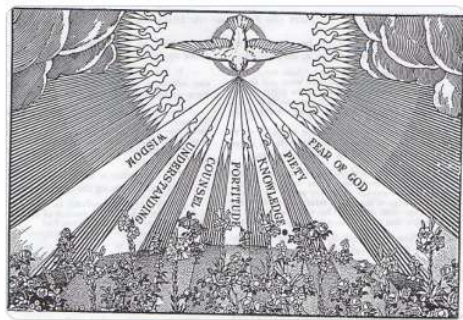

7:04 AM . Jan 6, 2017 - Twitter Web Client

2 Retweets 4 Likes

Q 0

(b)

Figure 3: @ChromHib tweets regarding "The Poems of Blathmac", stanza 6: translation and comments. Twitter threads respectively available via: a) https://twitter com/ChronHib/status/817249859874865157, and b) https://twitter.com/ ChronHib/status/817250468346798081 (accessed 2 July 2021). 
the transcription of Stanza 250 (see Figure 4). In three short comments the editor refers to differences in the texts of two previous editions. Further, he supplies the reader with details of the evolution of the editorial process and a justification of the new transcription and translation.

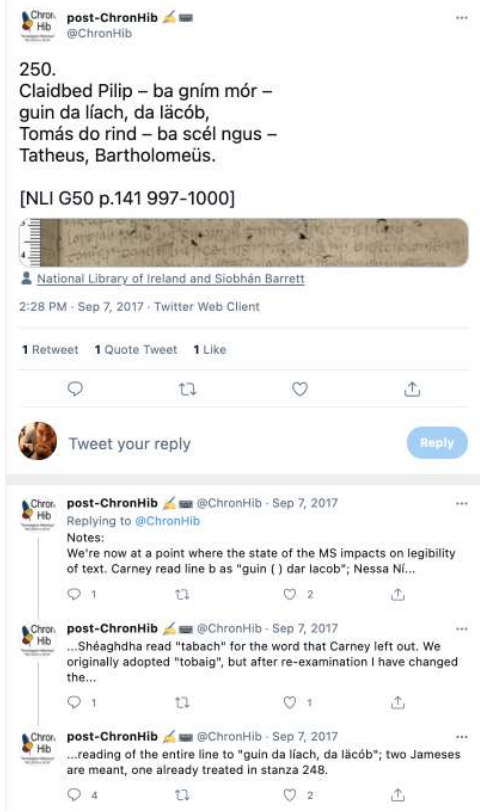

Figure 4: @ChromHib tweets regarding "The Poems of Blathmac", stanza 250: transcription and comments. ${ }^{\ddagger}$

In addition to editorial explanatory notes, the comments section provides an insight into user interaction. Indeed, the appropriateness of the term "user" in this context is questionable. The editor is often asked to provide explanations for details of the edition (see Figure 3a). Whilst the authority of the editor is taken for granted, the platform promotes a discourse between editor and commentators similar to that of a research seminar. Editing and translating Early Irish text is highly specialized and it is unsurprising that the majority of the non-editorial comments come from members of the scholarly community. In this way commentators are both contributors to and reviewers of the edited text.

$\ddagger \quad$ Twitter thread available via: https://twitter.com/ChronHib/status/9057 69726470754304 (accessed 2 July 2021). 


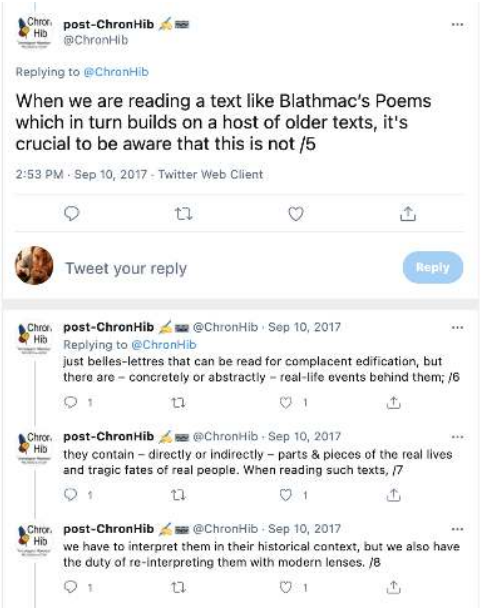

Figure 5: Twitter thread regarding images attached to the translation of stanza $253 . \S$

From the perspective of the reader (that is, the individual who may want to read and/or research the transcribed and translated stanzas sequentially), the structure of The Poems of Blathmac Tweets is far from ideal. Most notably, there is no hashtag categorizing the Tweets, making the series difficult to find and follow. This would be less problematic if the Twitter account were solely dedicated to the subject of the Poems of Blathmac. However, as previously observed, the account is extremely active and covers a broadly defined academic area. The absence of a hashtag is not insurmountable and can still be rectified by the creation of Twitter moments linking the Tweets but its current format presents barriers for those who wish to use it as a scholarly resource.

Whilst technically imperfect, The Poems of Blathmac Tweets uphold many of the tenets of digital scholarly editing and in particular of Web 2.0 and digital humanities as Humanities 2.0 as described in the previous section. The role of the tools of social media and social networking in the production of the resource is undeniable. The Tweets have attracted and activated the communities of both the scholarly and the wider public. However, the scholarly argument of the resource extends beyond the role of the tools of Web 2.0. They not only examine the historical context of the texts, they also encourage a view of the poems which highlights how modern audiences read and receive them. This is most successfully achieved through the inclusion of sometimes controversial modern iconography in the Tweets containing the translations of the stanzas. These images serve to enrich our reading of the text as the following excerpt from thread defending the decision to include an image of a young drowned

$\S \quad$ Twitter thread available via: https://twitter.com/ChronHib/status/9068 63322150129666 (accessed 2 July 2021). 
Syrian boy demonstrates (see Figure 5).

Not only do the Tweets blur the boundaries of many of the concepts of the hierarchical models of authority, such as editor, reader, user, contributor and reviewer, they go one step further, by allowing real-time comment and correction and through linking the contents of the poem to current affairs, they blur the temporal boundaries between then and now.

\section{IV}

Despite the functional deficiencies then, is it possible to argue that The Poems of Blathmac Tweets constitute a DSE? To answer this question, let us return to consider Sahle's broad definition of a digital scholarly edition and his differentiation between digitized print editions and digital editions. It is apparent that the series is not a digitized or scanned print edition, it does not represent the mere digitization of printed material, nor is it guided by a print paradigm. The Tweets cannot be printed without a loss of function: in their current form, they are extendable, open to comment and correction. Furthermore, as the preceding section demonstrates, many of the characteristics of the digital paradigm and the principles of social editing are exemplified in the creation of the Twitter series. Thus, at the very least we can assert that the Tweets represent a digital textual resource guided by a digital paradigm. If we continue to ask the four questions set forth by Sahle intended to establish a digital scholarly edition as such, we would find the answer to each question would be largely positive. Despite this, however, the Tweets fall short of Sahle's desideratum, and as such constitute an example of his vaguely described "other phenomena that are also related to the manifold activities in making our cultural heritage accessible" (Sahle 2016, 38).

In this context it is important to note a further aspect of Sahle's definition which is not detailed in the earlier discussion, notably that: "An edition project is not an edition" (Sahle 2016, 35). Here, he draws a line between a preliminary publication and an edition, which theoretically is always the best possible representation of the subject. The stated intention to use the project's Twitter feed as an outlet for a preliminary edition therefore greatly undermines its position as a DSE (see Figure 2). Despite claims that the digital editorial paradigm emphasizes the editorial process over the editorial product, the reality is that our means of assessment and evaluation are still product orientated: "Talking about editions, evaluating editions and cataloguing editions requires their identification by external boundaries and internal constituents" (Sahle 2016. 36). Definitions of DSEs are intended to be as exclusive as they are inclusive and no matter which criteria we apply The Poems of Blathmac Tweets fall short of the mark. To assess an edition there must be an edition, i.e., an editorial product.

In his highly-influential The Rationale of Hypertext, Jerome J. Mcgann wrote: "When we use books to study books, or hard copy texts to analyse other hard copy texts, the scale of the tools seriously limits the possible results" (McGann 2001). More recently Franzini et al. have noted that to date, the work undertaken 
by textual scholars to understand the impact of digital technologies on the interpretive study of texts has been generally approached from the perspective of the DSE, "and, as a result, there is no overarching understanding of how digital technologies have been employed across the full range of textual interpretations" (Franzini et al. 2016, 161). As we continue to assess the ideal of edition in the twenty-first century, it is important to consider how a continued emphasis on the edition may ultimately limit our understanding of how the digital medium is influencing textual scholarship. As Pierazzo comments: "At the present time, it seems that placing boundaries around the types of resources that can be produced might not be a productive way to look at the transformations introduced by the digital medium" (Pierazzo 2014, 210).

The Poems of Blathmac Twitter series was not intended as a digital edition, and many of its perceived shortcomings are as a result of this fact. However, to my mind, this is also one of the resource's strengths: free from the constraints of the label of a DSE, the project was able to explore the potential of social media in the creation of a scholarly edition. Reflecting on the terms we use to describe text-based digital scholarship, Kenneth Price concludes that we do not yet have a satisfactory term to describe this kind of work. Though Price's comments are concerned with the products of "large-scale" digital projects which have become the basic unit of digital humanities scholarship (Price 2009). A similar argument can be made around the products of small-scale digital textual research outputs. As we move towards a theory of digital scholarly editing which emphasizes process as well as product, we may ultimately have to move away from our product-oriented definitions of digital scholarly editions. This is particularly pertinent when we consider that the editorial products which scholars continue to employ in order to determine what constitutes a DSE may have been established according to a set of epistemologies which are no longer congruent with many of the aspirations of the contemporary editorial process.

\section{Bibliography}

1997-. "Celt: Corpus of Electronic Texts." Available from: https://celt.ucc.ie (Accessed: 2021-07-03).

Buzzoni, Marina, 2016. "A Protocol for Scholarly Digital Editions? The Italian Point of View." In Digital Scholarly Editing: Theories and Practices, edited by Elena Pierazzo and Matthew James Driscoll, Open Book Publishers, pages 59-82.

Davidson, CAthy, 2008. "Humanities 2.0: Promises, Perils, Predictions." PMLA 123(3), pages 707-17.

DiNucci, Darcy, 1999. “Fragmented Future.” Print 53(4), pages 221-22.

Doran, Michelle, 2015. Textual Criticism and Medieval Irish Studies. DPhil, University College Cork, Cork. 
, 2016. "Reflections on Medieval Studies and the Typology of Digital Scholarly Editions." Unpublished essay.

Edmond, Jennifer, ed., 2020. Digital Technology and the Practices of Humanities Research. Cambridge: Open Book Publishers. doi: https://doi.org/10.11647/OBP.0192.

Franzini, Greta, Melissa Terras, and Simon Mahony, 2016. "A Catalogue of Digital Editions." In Digital Scholarly Editing: Theories and Practices, edited by Elena Pierazzo and Matthew James Driscoll, Cambridge: Open Book Publishers, pages 161-82.

Kirschenbaum, Matthew, 2010. "What Is Digital Humanities and What's It Doing In English Departments?" Association of Departments of English (ADE) Bulletin 150. doi: https://doi.org/10.1632/ade.150.55

McGann, Jerome, 2001. The Rationale of Hypertext, New York (NY): Palgrave McMillan, pages 53-75.

Pierazzo, Elena, 2014. Digital Scholarly Editing: Theories Models and Methods. Abingdon-on-Thames: Routledge.

_ 2016. "Modelling Digital Scholarly Editing: From Plato to Heraclitus." In Digital Scholarly Editing: Theories and Practices, edited by Elena Pierazzo and Matthew James Driscoll, Cambridge: Open Book Publishers, pages 41-58.

Prescott, Andrew, 2003. "Riffs on McCarthy." Available from: http://digitalriffs.blogspot.com/2013/07/riffs-on-mccarty.html (Accessed: 2021-07-02).

Price, Kenneth M., 2009. “Edition, Project, Database, Archive, Thematic Research Collection: What's in a Name?" Digital Humanities Quarterly 3(3). Available from: http://www.digitalhumanities.org/dhq/vol/3/3/000053/000053.html (Accessed: 2021-07-02).

Robinson, Peter, 2005. “Current Issues in Making Digital Editions of Medieval Texts, or Do Electronic Scholarly Editions Have a Future?" Digital Medievalist 1. doi: http://doi.org/10.16995/dm.8.

, 2013. "Towards a Theory of Digital Editions." Variants, The Journal of the European Society for Textual Scholarship 10, pages 126-27.

2016a. "The Digital Revolution in Scholarly Editing." Ars Edendi Lecture Series IV pages 181-207. 
2016b. "Social Editions, Social Editing, and Social Texts." Digital Studies

/ Le Champ Numérique 6. Available from:

https://www.digitalstudies .org/articles/10.16995/dscn.6/

(Accessed: 2022-07-02).

SAhle, PAtrick, 2016. "What is a Digital Scholarly Edition." In Digital Scholarly Editing: Theories and Practices, edited by Elena Pierazzo and Matthew James Driscoll, Cambridge: Open Book Publishers, pages 19-40.

Schreibman, Susan, Ray Siemens, and Jon Unsworth, eds., 2014. A Companion to Digital Humanities. Oxford: Blackwell.

Siemens, Ray, Megan Timney, Cara Leitch, Corina Koolen, and Alex Garnett, 2012. "Toward Modeling the Social Edition: An Approach to Understanding the Electronic Scholarly Edition in the Context of New and Emerging Social Media." Literary and Linguistic Computing 27, pages 445-61.

Svensson, Patrick, 2009. "Humanities Computing as Digital Humanities." Digital Humanities Quarterly 3(3). Available from: https : //www.digitalhumanities.org/dhq/vol/3/3/000065/000065.html (Accessed: 2021-07-02).

2010. “The Landscape of Digital Humanities." Digital Humanities Quarterly 4(1). Available from: http://digitalhumanities.org/dhq/vol/4/1/000080/000080.html (Accessed: 2021-07-02).

2016. Big Digital Humanities: Imagining a Meeting Place for the Humanities and the Digital. Ann Arbor (MI: University of Michigan Press.

doi: https ://doi.org/10.2307/j.ctv65sx0t.12

TEI Consortium, 2020. “TEI P5: Guidelines for Electronic Text Encoding and Interchange." Available from: https://tei-c.org/release/doc/tei-p5-doc/en/Guidelines.pdf (Accessed: 2021-03-02).

VAn Zundert, Joris, 2016a. “Barely Beyond the Book?” In Digital Scholarly Editing: Theories and Practices, edited by Elena Pierazzo and Matthew James Driscoll, Cambridge: Open Book Publishers, pages 83-106.

- 2016b. "The Case of the Bold Button: Social Shaping of Technology and the Digital Scholarly Edition." Digital Scholarship in the Humanities 31(4), pages 898-910. doi: https://doi.org/10.1093/llc/fqw012

, 2018. "On Not Writing a Review about Mirador: Mirador IIIF, and the Epistemological Gains of Distributed Digital Scholarly Resources." Digital Medievalist 11, pages 5: 1-48. doi: http://doi.org/10.16995/dm.78 